tive Filterversuche genauer bestimmt werden. Da jedoch das Isotopenbild des $\mathrm{Yb}$ keinen weiteren K-Strahler außer Yb169 (33 d) zuläßt, muß diese K-Strahlung mit dem $\beta$-Zerfall des Yb $(99 \mathrm{~h})$ zusammenhängen. Die einfachste Deutung ist folgende. Beim $\beta$-Zerfall des $Y b(99 \mathrm{~h})$ werden teilweise angeregte Zustände von durchschnittlich etwa $350 \mathrm{ekV}$ gebildet. Die hieraus entstehende $\gamma$-Strahlung stellt z. Tl. die harte Komponente dar, z. Tl. erleidet sie innere Umwandlung in der K-Schale, so daß Cp-KStrahlung entsteht. Hiermit sind folgende Tatsachen im Einklang. Die K-Strahlung ist vergleichsweise schwach. Die Energiedifferenz der beiden $\beta$-Gruppen (500-130 ekV; Ziff. 7) stimmt ungefähr mit der gemessenen $\gamma$-Energie überein. Koinzidenzmessungen von H. Maier-Leibnitz zeigten, daß diese $\gamma$-Strahlung mit der $130-\mathrm{ekV}$ Komponente, nicht aber mit der 500-ekV-Kom- ponente der $\beta$-Strahlung gekoppelt ist. Die Aussendung der 500-ekV-Elektronengruppe führt also unmittelbar zum Grundzustand des Endkerns.

10. Die kurzlebige Yb-Aktivität. Zuordnungen. Ein kurzer Versuch mit schwacher Bestrahlung ergab eine weitere $\mathrm{Yb}$-Aktivität von 2,4 $\pm 0,2 \mathrm{~h}$, was mit früheren Angaben ${ }^{14}$ in Einklang ist. Die Absorptionskurve läßt auf eine Grenzenergie von schätzungsweise $1,3 \mathrm{eMV}$ schließen. Dagegen konnte für eine von $\mathrm{P}$ o ol u. Quill (l. c. ${ }^{14}$ ) angegebene 41-h-Aktivität keine Andeutung gefunden werden.

Es sind somit drei radioaktive Yb-Isotope einzuordnen. Der K-Strahler von $33 \mathrm{~d}$ kann nur $\mathrm{Yb}^{\mathbf{1 6 9}}$ sein. Für die $\beta$-Strahler von $2,4 \mathrm{~h}$ und $99 \mathrm{~h}$ kommen dann nur die Plätze $\mathrm{Yb}^{175}$ oder $\mathrm{Yb}^{177}$ in Betracht.

14 G. Hevesy u. H. Levi ${ }^{3}$; N. L. Pool u. L. I. Quill, Physic. Rev. 53, 437 [1938].

\title{
Die Aktivierung der seltenen Erden durch thermische Neutronen II $^{1}$
}

\author{
Von Walther Bothe
}

Aus dem Institut für Physik am Kaiser-Wilhelm-Institut für medizin. Forscḩung, Heidelberg

(Z. Naturforschg. 1, 179-189 [1946]; eingegangen am 14. Dezember 1945)

Entsprechende Untersuchungen, wie früher für Tm, Yb und $\mathrm{Cp}$ beschrieben ${ }^{2}$, wurden an folgenden Elementen angestellt: Sc, Y, La, Ce, Pr, Nd, Sm, Eu, Tb, Dy, Ho, Er. Die erhaltenen Aktivitäten sind in Tab. 1 verzeichnet. Praktische Intensitätsangaben und rohe Werte der Aktivierungsquerschnitte mit thermischen Neutronen für alle Erden (außer Gd) finden sich am Schluß in Tab. 2.

1. Scandium und Ytrium. Diese beiden Elemente mußten in die Untersuchung einbezogen werden, weil sie als Verunreinigungen der seltenen Erden in Betracht kommen. Ein kräftig mit thermischen Neutronen bestrahltes Präparat von $\mathrm{Y}_{2} \mathrm{O}_{3}$ (,rein bis auf $0,2 \% \mathrm{Ho}+\mathrm{Er}^{\text {, }}$, wozu noch $0,05 \%$ Dy gefunden wurde) zeigte einheitlichen Abfall bis zu einigen $1^{-5}$ der Anfangsaktivität. Als Halbwertzeit des $\mathrm{Y}^{90}$ ergab sich $\mathrm{T}=61 \pm 1 \mathrm{~h}$, in Übereinstimmung mit dem von D. W. Stewart, J. L. Law s on und J. M. C o r k $\mathbf{k}^{3}$ angegebenen Wert (60,5 $\pm 2 \mathrm{~h})$. Die Absorptionskurve der. Elektronen zeigt Abb. 1. Die $\beta$-Grenze liegt nach anderwei-

1 Dieser II. Teil wurde (in etwas ausführlicherer Fassung) im September 1944 der Z. Physik eingereicht, kam aber bisher nicht zur Veröffentlichung.

2 Diese Z. 173-179 (im folgenden als I angeführt).

3 Physic. Rev. 52, 901 [1937].

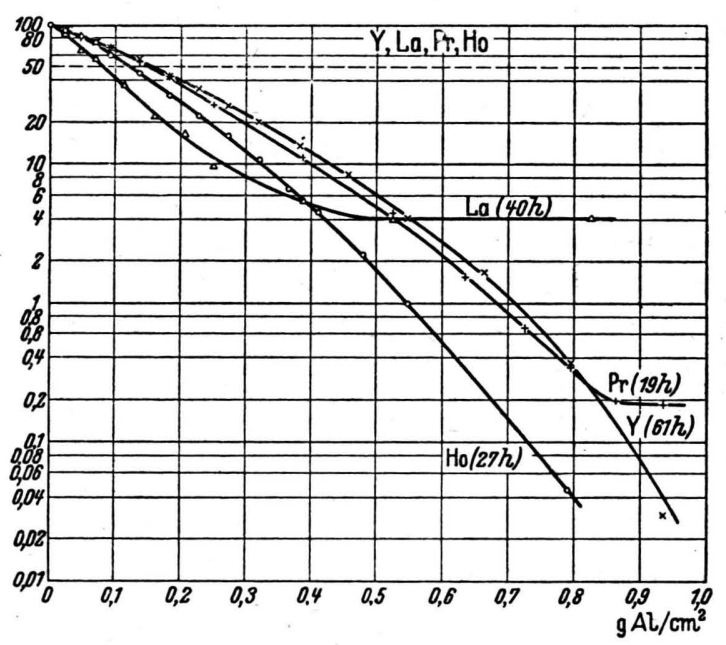

Abb. 1. Absorptionskurven der Y-, La-, Pr- und Ho-Strahlungen in Aluminium.
Dieses Werk wurde im Jahr 2013 vom Verlag Zeitschrift für Naturforschung in Zusammenarbeit mit der Max-Planck-Gesellschaft zur Förderung der Wissenschaften e.V. digitalisiert und unter folgender Lizenz veröffentlicht: Creative Commons Namensnennung-Keine Bearbeitung 3.0 Deutschland Lizenz.

Zum 01.01.2015 ist eine Anpassung der Lizenzbedingungen (Entfall der Creative Commons Lizenzbedingung „Keine Bearbeitung“) beabsichtigt, um eine Nachnutzung auch im Rahmen zukünftiger wissenschaftlicher Nutzungsformen zu ermöglichen.
This work has been digitalized and published in 2013 by Verlag Zeitschrift für Naturforschung in cooperation with the Max Planck Society for the Advancement of Science under a Creative Commons Attribution-NoDerivs 3.0 Germany License.
On 01.01.2015 it is planned to change the License Conditions (the removal of the Creative Commons License condition "no derivative works"). This is to allow reuse in the area of future scientific usage. 


\begin{tabular}{|c|c|c|c|c|c|c|}
\hline Aktives Isotop & Halbwertzeit & $\begin{array}{l}\text { Zer- } \\
\text { fall }\end{array}$ & $\beta$-Str. eMV & $\gamma$-Str. & K-Str. von & Zerfallsprodukt \\
\hline $\mathrm{Y}^{90} \ldots \ldots \ldots \ldots$ & $61 \pm 1 \mathrm{~h}$ & $\beta$ & $\left.2,1-2,6^{*}\right)$ & - & - & $\mathrm{Zr}^{90}$ \\
\hline $\mathrm{La}^{140} \ldots \ldots \ldots$ & $39,5 \pm 0,5 \mathrm{~h}$ & $\beta$ & 1,3 & $\left.0,9 \mathrm{eMV}^{7}\right)$ & - & $\mathrm{Ce}^{140}$ \\
\hline 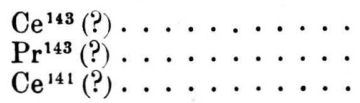 & $\begin{array}{r}33 \pm 2 \mathrm{~h} \\
12,5 \pm 1 \mathrm{~d} \\
30 \pm 2 \mathrm{~d}\end{array}$ & $\beta_{\beta}$ & $\begin{array}{l}1,3 \\
0,9 \\
0,4\end{array}$ & $\begin{array}{l}? \\
? \\
+\end{array}$ & - & $\begin{array}{l}\operatorname{Pr}^{143}(?) \\
\operatorname{Nd}^{143}(?) \\
\operatorname{Pr}^{141}(?)\end{array}$ \\
\hline $\operatorname{Pr}^{142} \ldots \ldots \ldots$ & $19,2 \pm 0,3 \mathrm{~h}$ & $\beta$ & 2 & schwach? & schwach? & $\mathrm{Nd}^{142}$ \\
\hline $\begin{array}{l}\mathrm{Nd}^{147},{ }^{119},{ }^{151} \text { oder } \\
61^{147},{ }^{149},{ }^{151} \\
\left(\text { oder }^{*} \mathrm{Sm}^{151}\right)\end{array}$ & $\begin{array}{l}120 \pm 3 \mathrm{~m} \\
47,5 \pm 1,5 \mathrm{~h} \\
11,1 \pm 0,2 \mathrm{~d}\end{array}$ & $\begin{array}{l}\beta \\
\beta \\
\beta\end{array}$ & $\begin{array}{l}1,6 \\
1,1 \\
0,8\end{array}$ & $\begin{array}{l}? \\
? \\
?\end{array}$ & - & $\left\{\begin{array}{l}61^{147},{ }^{149},{ }^{151} \\
\mathrm{Sm}^{147},{ }^{149},{ }^{151} \\
\left(\text { oder } \mathrm{Eu}^{151}\right)\end{array}\right.$ \\
\hline $\mathrm{Sm}^{151},{ }^{153},{ }^{155}\left(\right.$ oder $\left.{ }^{*} \mathrm{Eu}^{155}\right)$ & $47 \pm 1 \mathrm{~h}$ & $\beta$ & 0,75 & - & Eu (inn. Umw.) & $\mathrm{Eu}^{151},{ }^{153},{ }^{155}\left(\mathrm{od} . \mathrm{Gd}^{155}\right)$ \\
\hline$E_{152 \quad 154}$ & $9,3 \pm 0,2 \mathrm{~h}$ & $\beta_{\mathrm{K}}$ & $\frac{1,8}{-}$ & $\stackrel{+}{?}$ & $\overline{\mathrm{Sm}}$ & $\begin{array}{l}\mathrm{Gd}^{152},{ }^{154} \\
\mathrm{Sm}^{152},{ }^{154}\end{array}$ \\
\hline $\mathrm{Eu}$ & $7 \pm 4 a$ & $\stackrel{\beta}{\mathrm{K}}$ & $\underline{1,0}$ & $\begin{array}{l}? \\
+\end{array}$ & $\overline{\mathrm{Sm}}$ & $\begin{array}{l}\mathbf{G d}^{152},{ }^{154} \\
\mathrm{Sm}^{152},{ }^{154}\end{array}$ \\
\hline $\mathrm{Tb}^{160} \ldots \ldots$ & $73,5 \pm 1 d$ & $\beta$ & 0,75 & + & $?$ & $\mathrm{Dy}^{160}$ \\
\hline $\mathrm{Dy}^{165} \ldots \ldots \ldots$ & $140 \pm 1,5 \mathrm{~m}$ & $\beta$ & $\left.1,4-1,9^{*}\right)$ & - & - & $\mathrm{Ho}^{165}$ \\
\hline $\mathrm{Ho}^{166} \ldots \ldots \ldots$ & $27,3 \pm 0,5 \mathrm{~h}$ & $\beta$ & 1,8 & - & - & $\operatorname{Er}^{166}$ \\
\hline $\operatorname{Er}^{169},{ }^{171}\left(\right.$ oder $\left.\operatorname{Tm}^{171}\right) \cdot$ & $\begin{aligned} & \sim 6 \mathrm{~m} \\
5,7 & -7,1 \mathrm{~h} \\
20 & \pm 2 \mathrm{~h}\end{aligned}$ & $\begin{array}{l}\beta \\
\beta \\
\beta\end{array}$ & $\begin{array}{l}? \\
1,6 \\
0,6\end{array}$ & $\begin{array}{l}? \\
+ \\
+\end{array}$ & $\frac{?}{-}$ & $\mathrm{Tm}^{169},{ }^{171}\left(\mathrm{od} . \mathrm{Yb}^{171}\right)$ \\
\hline
\end{tabular}

*) Vgl. J. M a tt a u ch und S. Flügge, Kernphysikal. Tabellen, Berlin 1942.

Tab. 1. Die bei der Bestrahlung seltener Erden mit thermischen Neutronen auftretenden Kernreaktionen und Halbwertszeichen.

tigen Messungen etwas über 2 eMV. Eine $\gamma$-Strahlung tritt nicht auf.

Scandium ist bereits von $\mathrm{H}$. W a l $\mathrm{ke}^{4}$ sorgfältig untersucht worden. Soweit unsere Messungen an

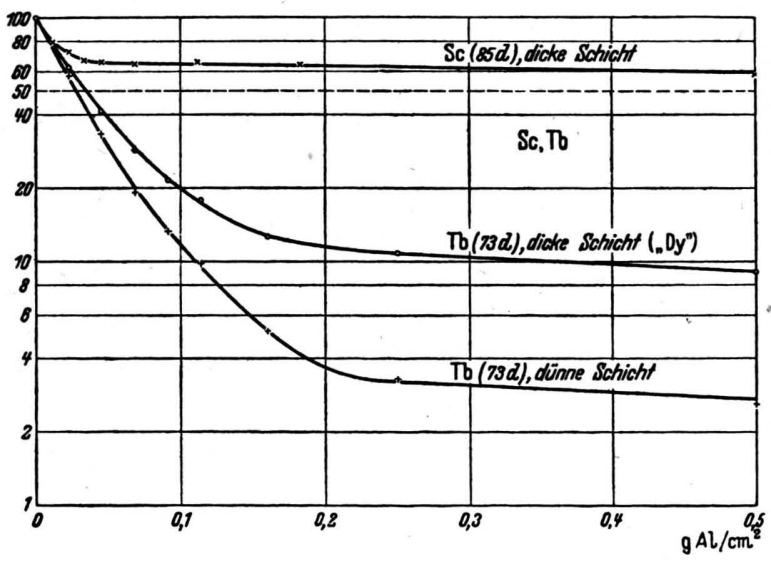

Abb. 2. Absorptionskurven der Sc- und Tb-Strahlungen in Aluminium.

$\mathrm{Sc}_{2} \mathrm{O}_{3}$,reinst" reichen, geben sie dieselbe Halbwertzeit von $85 \mathrm{~d}$ für $\mathrm{Sc}^{46}$. Für die Absorptions-

4 Physic. Rev. 57, 163 [1940].

5 Nature 136, 102 [1935]. kurve (Abb.2) ist kennzeichnend das geringe Durchdringungsvermögen der $\beta$-Strahlung (die hier schon zum größten Teil in der Zählrohrwand stecken bleibt) und die intensive $\gamma$-Strahlung.

2. Lanthan. $\mathrm{La}_{2} \mathrm{O}_{3}$ zeigte nach kräftiger Bestrahlung mit thermischen Neutronen einheitlichen $A b$ fall, der bis $\mathrm{zu}<10^{-3}$ der Anfangsaktivität verfolgt wurde. Die Halbwertzeit des entstehenden $\mathrm{La}^{140}$ ist $\mathrm{T}=39,5 \pm 0,5 \mathrm{~h}$. Die von J. K. M a rsh u. S.Sugden ${ }^{5}$ und von Götte ${ }^{6}$ gemessenen Werte von 46 bzw. $44 \pm 2 \mathrm{~h}$ sind sicher zu hoch. In Bestätigung unseres Wertes hat kürzlich W. Ma u re r ${ }^{7}$ aus La ${ }^{139}(d, p) L^{140}$ den Wert von $40 \pm 1,5 \mathrm{~h}$ gefunden. Die Absorptionskurve der Elektronen zeigt Abb.1. Sie läßt auf eine Grenzenergie von etwa 1,3 eMV schließen. Nach der Kurvenform dürfte jedoch die $\beta$-Strahlung aus mehreren Gruppen von verschiedener Grenzenergie zusammengesetzt sein. Dementsprechend ist auch eine $\gamma$-Strahlung vorhanden. Die $\gamma$-Strahlung einer dünnen Schicht macht rd. 2\% der $\beta$-Ausschläge, wobei letztere für die Absorption in der Zählrohrwand kor-

6 Vergl. O. Hahn u. F. Stra ß mann, Naturwiss. 28, 543 [1940].

7 unveröffentlicht. 
rigiert sind. Die Energie der $\gamma$-Strahlung schätzt M a u rer aus der Absorption zu ungefähr 0,9 eMV. Aus diesen Daten kann mit Berücksichtigung der Ansprechwahrscheinlichkeit des Zählrohres ${ }^{8}$ geschlossen werden, daß mehr $\gamma$-Strahlen als $\beta$-Strahlen entstehen, es sei denn, daß noch eine sehr weiche Gruppe von $\beta$-Strahlen vorhanden ist, die nicht in das Zählrohr dringt. Dies alles deutet darauf hin, daß beim Zerfall des $\mathrm{La}^{140}$ überwiegend angeregte $\mathrm{Ce}^{140}$-Kerne entstehen, die stufenweise in den Grundzustand übergehen.

3. Cer. Sehr kräftige Bestrahlung von $\mathrm{CeO}_{2}$ („reinst, von anderen Erden frei“) mit thermischen Neutronen ergab die in Abb. 3 wiedergegebene in Paraffin mit Cadmium bedeckt, so gingen alle drei Aktivitäten auf wenige Prozente herunter. Sie entstehen also weit überwiegend durch thermische Neutronen.

Chemische Trennungsversuche an bestrahltem Cer konnten leider nicht mehr bis zu einem voll befriedigenden Ergebnis durchgeführt werden. Es scheint aber danach nicht ausgeschlossen, daß die 12,5-d-Aktivität zu einem anderen Element als Ce gehört. Die einfachste Deutung wäre dann:

$$
\mathrm{Ce}^{143}(33 \mathrm{~h}) \longrightarrow \operatorname{Pr}^{143}(12,5 \mathrm{~d}) \longrightarrow \mathrm{Nd}^{143} .
$$

Für diese Deutung spricht besonders, daß die nach Ziff. 13 formal berechneten „Aktivierungsquer-

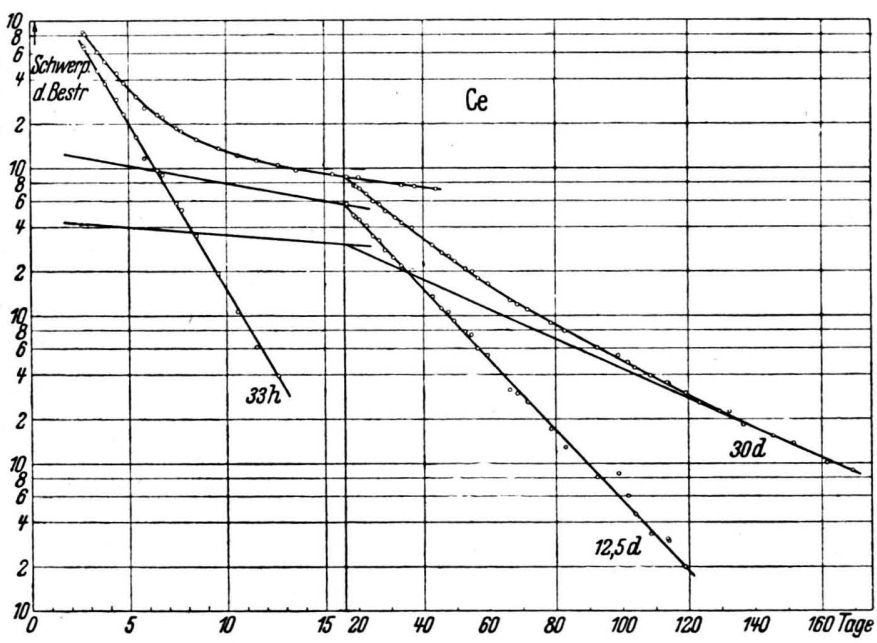

Abb. 3. Abfallskurve von Ce.

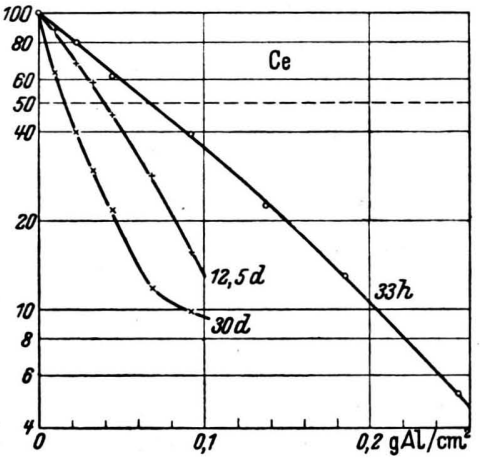

Abb. 4. Absorptionskurven der Ce-Strahlungen in Aluminium.
Abfallskurve. Die Aktivitäten waren schwach. Die Kurvenanalyse führt auf drei vorher nicht bekannte Körper mit den Halbwertzeiten $33 \pm 2 \mathrm{~h}$; $12,5 \pm 1 \mathrm{~d} ; 30 \pm 2 \mathrm{~d}$. Die Absorptionskurven der Strahlung in Aluminium wurden in den verschiedenen Stadien des Abfalls aufgenommen und daraus die Absorptionskurven für die drei Komponenten abgeleitet (Abb.4). Die Kurven für die beiden langlebigsten Komponenten sind weniger genau. Aus diesen Absorptionskurven kann man die drei Grenzenergien grob schätzen auf 1,$3 ; 0,9 ; 0,4 \mathrm{eMV}$ für den 33-h-, 12,5-d-, 30-d-Körper. Der 30-d-Körper scheint eine aus mehreren Teilspektren zusammengesetzte $\beta$-Strahlung und eine intensive $\gamma$-Strahlung zu haben.

Wurde das Präparat während der Bestrahlung

8 F. Norling, Ark. Mat., Astron., Fysik, Ser. A 27, 1 [1941]; H. Ma i e r - Le ibn it z, erscheint demnächst. schnitte" für die beiden Aktivitäten nahezu gleich sind (Tab. 2, Spalte 5); dies ist zu verlangen, wie eine einfache Rechnung zeigt. Die 30-d-Aktivität würde dann dem $\mathrm{Ce}^{141}$ zuzuschreiben sein.

Eine von Ruben u. Kamen ${ }^{9}$ angegebene $\beta$-Strahlung von nur $0,12 \mathrm{eMV}$ und $\mathrm{T}=15 \mathrm{~d}$ konnte auch mit einem dünnwandigen Glimmer-Zählrohr nicht gefunden werden.

4. Praseodym. $\operatorname{Pr}_{4} \mathrm{O}_{7}$, zuverlässig als ,sehr rein" bezeichnet, zeigte mit thermischen Neutronen nur die bekannte 19-h-Aktivität des $\operatorname{Pr}^{142}$. Die Abfallskurve ergab als Halbwertzeit 19,2 $\pm 0,3 \mathrm{~h}$. Die Absorptionskurve der Strahlung zeigt Abb. 1. Nach der Reichweite liegt die Energiegrenze der $\beta$-Strahlen nahe bei 2 eMV. Eine schwache $\gamma$ - oder K-Strahlung könnte neben der zu erwartenden Bremsstrahlung vorhanden sein.

Mit schnellen Neutronen (ohne Paraffin, Prä- 
parat in Cadmium eingeschlossen) konnte bisher ebenfalls nur die 19,2-h-Aktivität mit derselben Absorptionskurve beobachtet werden, und zwar in beachtlicher Intensität. Da Pr ein Reinelement ist, kann auch hier nur der $(n, \gamma)$-Prozeß, nicht ein

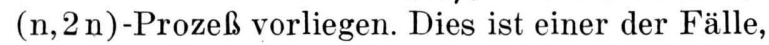
die zeigen, daß ein aktives Isotop keineswegs zwischen zwei stabilen liegen muß, wenn es sowohl durch thermische wie durch schnelle Neutronen entsteht - eine Schlußweise, die in der amerikanischen Literatur anzutreffen isf.

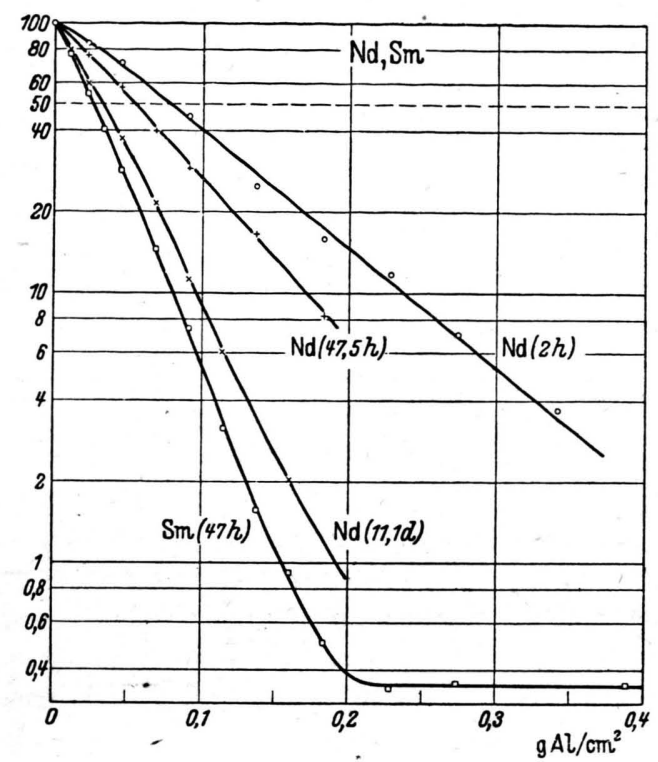

Abb. 5. Absorptionskurven der Nd- und Sm-Strahlungen. in Aluminium.

5. Neodym. $\mathrm{Nd}_{2} \mathrm{O}_{3}$,sehr rein" ergab in mehreren Versuchsreihen drei gut definierte Aktivitäten von $\mathrm{T}=120 \pm 3 \mathrm{~min} ; 47,5 \pm 1,5 \mathrm{~h}$ und $11,1 \pm 0,2 \mathrm{~d}$. Die beiden längerlebigen haben ähnliche Intensität wie die des Cers. Die Absorptionskurven der Strahlen wurden in verschiedenen Phasen des Abfalls gemessen und daraus die Absorptionskurven der drei Komponenten berechnet (Abb.5). Nach den Halbwertdicken kann man die Grenzenergien grob schätzen auf 1,$6 ; 1,1 ; 0 ; 8$ eMV für den 2-h-, 47-hund 11-d-Körper. Man sieht aus Abb.5, daß der 47-h-Körper sich in der Absorption deutlich vom $\mathrm{Sm}(47 \mathrm{~h})$ unterscheidet. Ebenso wenig kann der 11-d-Körper mit Ce $(12,5 \mathrm{~d})$ verwechselt werden (vergl. Abb.4).

Die 47-h- und 11-d-Aktivitäten wurden auch mit

9 Vergl. J. J. Livingood u. G. T. Se aborg, Rev. mod. Physics 12, 30 [1940]. schnellen Neutronen gefunden (nach der 2-h-Aktivität wurde nicht gesucht). Das Intensitätsverhältnis war jedoch verschieden. Während mit thermischen Neutronen die Sättigungsaktivität des 47-h-Körpers, korrigiert für Absorption im Präparat selbst und im Zählrohr, 0,9-mal so groß war wie die des 11-d-Körpers (Ziff. 13), betrug dieses Verhältnis mit schnellen Neutronen 2,2.

H. B. L a w, M. L.P o o l, J.D. K u r b a t ow u. L. L. Quill $\mathbf{1}^{\mathbf{1 0}}$ haben mit Neutronen und Deuteronen Aktivitäten von 2,3 h; $47 \mathrm{~h}$ und $10 \mathrm{~d}$ beobachtet, die mit den hier angegebenen sicher identisch sind.

Die elektronenstrahlenden Isotope des Nd sind besonders interessant, weil sie in das Element 61 übergehen, das höchstwahrscheinlich keine stabilen Isotope besitzt, so daß weiterer Zerfall nach Sm, unter Umständen sogar bis zum Eu zu erwarten ist. Aus diesem Grunde ist aber auch eine $\mathrm{Zu}$ ordnung der beobachteten Halbwertzeiten vorerst nicht möglich. Sie können entweder zu Nd-Isotopen gehören, oder zum Element 61, indem nämlich zunächst ein relativ kurzlebiges Nd-Isotop entsteht, das dann in ein 61-Isotop der beobachteten Halbwertzeit übergeht. Intensitätsmäßig wäre z. B. der 2-h-Körper als Mutter einer der beiden anderen Aktivitäten möglich (Tab.2). Selbst die folgende längere Zerfallsreihe ist nicht ganz auszuschließen :

$$
\mathrm{Nd}^{150}(\mathrm{n}, \gamma) \mathrm{Nd}^{151}(\mathrm{kurz}) \rightarrow 61^{151}(\mathrm{kurz}) \rightarrow \mathrm{Sm}^{151} \rightarrow \mathrm{Eu}^{151} \text {. }
$$

Das hypothetische $\mathrm{Sm}^{151}$ mit einer der hier beobachteten Aktivitäten konnte zwar bisher nicht aus $\mathrm{Sm}^{150}(\mathrm{n}, \gamma)$ gewonnen werden, es könnte aber isomer sein mit einer der beiden beim $\mathrm{Sm}$ beobachteten Aktivitäten, auch mit der noch fehlenden dritten Sm-Aktivität (vergl. Ziff. 6).

Der von J. C. McLennan u. W. H. Rann ${ }^{11}$ angegebene 35-h-Körper dürfte im wesentlichen unser 47-h-Körper sein. Eine von P o ol u. Quill ${ }^{12}$ angegebene 84-h-Aktivität wurde nicht beobachtet.

6. Samarium. Das Sm-Mg-Nitrat, aus dem das $\mathrm{Sm}_{2} \mathrm{O}_{3}$ hergestellt wurde, sollte 99,8-proz. sein mit Eu und Gd als Verunreinigungen: Die Abfallskurve nach Bestrahlung mit thermischen Neutronen wurde sowohl mit unbedecktem Präparat als auch mit $1 \mathrm{~mm}$ Al-Filter aufgenommen. Im letzteren Falle machte sich der Eu-Gehalt besonders deutlich durch die Abfalls- und Absorptionskurve bemerkbar, was nach den Absorptionsverhältnissen

\footnotetext{
10 Physic. Rev. 59, 936 [1941].

11 Nature 136, 831 [1935].

12 Physic. Rev. 53, 437 [1938].
} 
leicht verständlich ist. Im übrigen zeigten beide Kurven den bekannten 47-h-Abfall des Samariums; gemessen wurde $\mathrm{T}=47 \pm 1 \mathrm{~h}$. Dieselbe Aktivität wurde auch mit schnellen Neutronen erhalten. Die zugehörige Absorptionskurve ist in Abb. 5 eingetragen. Die Grenzenergie der $\beta$-Strahlung ist nach der Reichweite auf 0,75 eMV zu schätzen.

Die durchdringende Strahlung, die hinter $1 \mathrm{~mm}$ Al übrigbleibt, fällt ebenfalls mit $\mathrm{T}=47 \mathrm{~h}$ ab, gehört also zu demselben Isotop wie die $\beta$-Strahlung. Abb. 6 zeigt ihre Absorptionskurve in Blei. Diese läßt zwei Komponenten erkennen, von denen die härtere nach grober Schätzung eine mittlere Energie von etwa $0,3 \mathrm{eMV}$ hat. Die absolute Intensität

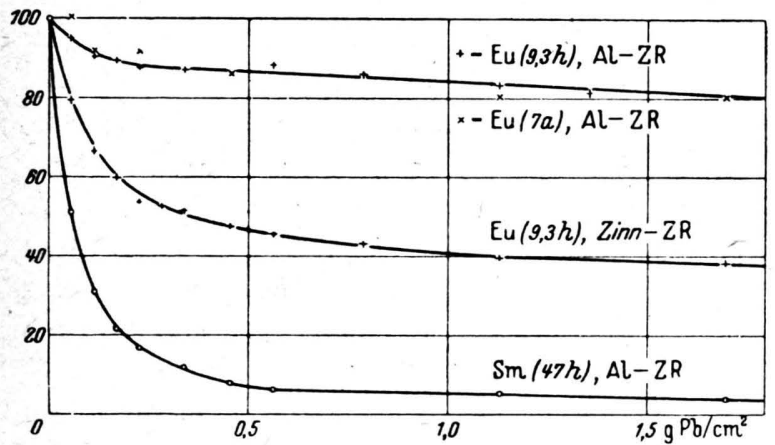

Abb. 6. Absorptionskurven der durchdringenden Sm- und Eu-Strahlungen in Blei.

dieser $\gamma$-Strahlung beträgt nur einige Quanten auf $100 \beta$-Teilchen, sie ist also als Bremsstrahlung der $\beta$-Strahlen zu verstehen. Die Absorbierbarkeit der weichen Komponente läßt sogleich eine K-Strahlung vermuten. Um dies zu prüfen, wurden $\mathrm{Ab}$ sorptionsmessungen mit selektiven Filtern gemacht, wie in I beschrieben. Der Absorptionssprung wurde zwischen Ce und Pr gefunden. Es liegt also die K-Strahlung des Folgeproduktes Eu vor. Hiernach ist der Ursprung dieser K-Strahlung klar: das Sm geht unter $\beta$-Zerfall in ein angeregtes Eu über, dessen $\gamma$-Strahlung in der K-Schale umgewandelt wird und.zwar praktisch vollständig. Der $\gamma$-Übergang im Eu muß daher hoch verboten sein, das angeregte Eu kann als metastabil angesprochen werden; seine Halbwertzeit kann jedoch nicht größer als $47 \mathrm{~h}$ sein. Es ist ein Isomer entweder eines der beiden stabilen Eu-Kerne $\left(\mathrm{Eu}^{151},{ }^{153}\right)$ oder des noch unbekannten $\mathrm{Eu}^{\mathbf{1 5 5}}$.

Interessant sind in diesem Falle noch die Intensitätsverhältnisse. Da der $\gamma$-Übergang vom metastabilen zum normalen $\mathrm{Eu}$ hoch verboten ist, wird -wahrscheinlich dasselbe für den direkten $\beta$-Übergang vom Sm zum normalen Eu gelten, es wird also praktisch nur der $\beta$-Übergang zum metastabilen Eu auftreten. Daher sollten ebenso viele primäre $\beta$-Teilchen wie K-Anregungen vorkommen. Weiter entstehen aber bei der inneren Umwandlung der $\gamma$-Strahlen noch sekundäre $\beta$-Teilchen in derselben Zahl wie die primären. Wahrscheinlich werden jedoch die sekundären $\beta$-Teilchen so weich sein, daß sie nur zum Teil durch die Messung und die Absorptionskorrektionen erfaßt werden. Jedenfalls ist hiernach für das beobachtete Verhältnis von $\mathrm{K}$-Anregungen zu $\beta$-Teilchen ein Wert zwischen $1 / 2$ und $1 \mathrm{zu}$ erwarten, wenn man die Korrektionen für Absorption im Präparat selbst und in der Zählrohrwand in der in Ziff. 13 beschriebenen Weise ausführt. Nach Tab. 2 ist der tatsächlich gefundene Wert 0,7 , in Übereinstimmung mit der Erwartung.

Der Vollständigkeit wegen sei noch eine andere Deutungsmöglichkeit erwähnt. Die Halbwertzeit von $47 \mathrm{~h}$ könnte zu dem metastabilen Eu selbst gehören, das dann aus einem kurzlebigen Sm entstehen müßte. Die primären $\beta$-Strahlen würden dann fortfallen, und es müßten ebenso viele K-Anregungen wie $\beta$-Teilchen auftreten. Auch sind dann die beobachteten $\beta$-Teilchen wohl energiereicher, als man nach den bei anderen Isomerenpaaren vorkommenden Energiedifferenzen erwarten würde. Diese Schwierigkeiten fallen allerdings fort, wenn man als Produkt das sicher radioaktive $\mathrm{Eu}^{155}$ annimmt:

$$
\mathrm{Sm}^{155}(\mathrm{kurz}) \rightarrow{ }^{*} \mathrm{Eu}^{155}(47 \mathrm{~h}) \rightarrow \mathrm{Eu}^{155}(\mathrm{kurz}) \rightarrow \mathrm{Gd}^{155} .
$$

Die beobachteten $\beta$-Teilchen wären dann im wesentlichen die des $\mathrm{Eu}^{155}$, und die Intensitätsverhältnisse wären im Endeffekt dieselben wie bei der ersten Deutung. Vorläufig liegt jedoch kein Grund vor, diese zweite Deutung vorzuziehen.

Die kurzlebige Sm-Aktivität von $\mathrm{T} \approx 1 / 2 \mathrm{~h}$, die Fermi und Hevesy u. Levi sowie Pool u. Quill angeben, wurde hier ebenfalls beobachtet, aber nicht genauer untersucht.

Eine sichere Zuordnung der beiden Aktivitäten $\mathrm{zu}$ den drei möglichen Isotopen $\mathrm{Sm}^{151,153,155}$ ist nach dem obigen nicht ohne weiteres möglich.

7. Europium. Für das benutzte $\mathrm{Eu}_{2} \mathrm{O}_{3}$ waren als einzige Verunreinigungen angegeben : $0,03-0,04 \%$ Gd und $0,02-0,03 \% \mathrm{Sm}$. Es wurden nur die beiden schon bekannten Eu-Aktivitäten beobachtet. Für die kurzlebige wurde $\mathrm{T}=9,3 \pm 0,2 \mathrm{~h}$ gemessen. 
Diese Aktivität ist sehr stark und kann daher zum Nachweis sehr geringer Mengen von Eu dienen ${ }^{13}$. Sie wird in der Tat in den meisten Erdenpräparaten vom Sm bis zum Dy gefunden. Für die längere der beiden Halbwertzeiten konnte auch hier zunächst nur eine untere Grenze von 3 a festgestellt werden. Weitere Schlüsse auf diese Halbwertzeit werden sich jedoch aus den weiter unten diskutierten Intensitätsverhältnissen ergeben.

Die Absorptionskurven der beiden $\beta$-Strahlungen zeigt Abb. 7. Die Grenzenergien liegen hiernach bei rd. 1,8 bzw. 1,0 eMV für die 9,3-h- bzw. die langlebige Aktivität, in Übereinstimmung mit an-

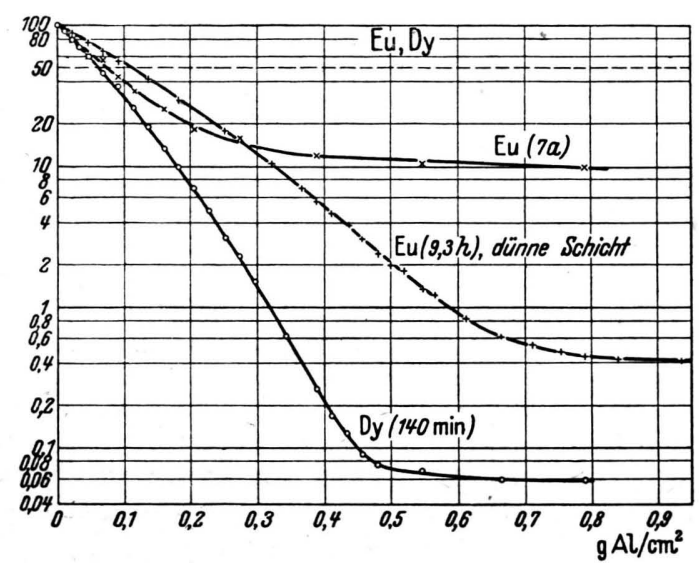

Abb. 7. Absorptionskurven der Eu- und Dy-Strahlungen in Aluminium.

derweitigen Messungen ${ }^{14}$. Beide Aktivitäten sind mit einer durchdringenden Strahlung verbunden, die bei der langlebigen Aktivität außergewöhnlich stark ist. In Abb. 6 sind die Blei-Absorptionskurven der durchdringenden Strahlungsanteile nach Wegfilterung der $\beta$-Strahlen durch Aluminium dargestellt. Die beiden Kurven decken sich praktisch und zeigen neben echten $\gamma$-Strahlen auch Andeutungen von K-Strahlung.

H. Maier-Leibnitz hat ein Zählrohr mit Zinnauskleidung $\left(10 \mathrm{mg} / \mathrm{cm}^{2}\right)$ konstruiert, das besonders empfindlich für K-Strahlen dieses Bereiches ist, und das er dem Verf. freundlichst zur Verfügung stellte. Die damit aufgenommene Absorptionskurve für die 9,3-h-Aktivität ist ebenfalls in Abb. 6 eingetragen. Diese läßt nun die K-Strahlung sehr deutlich erkennen. Versuche mit selektiven Filtern wurden entsprechend wie beim Sm ausge-

13 G. Hevesy u. H. Levi, Danske Videnskab. Selskab. XIV, Nr. 5 [1936] und XV, Nr. 11 [1938]. führt. Der Absorptionssprung wurde jetzt $\mathrm{zwi}$ schen La und $\mathrm{Ce}$ gefunden. Die vorliegende $\mathrm{K}$-Strahlung ist also die des $\mathrm{Sm}$; sie entsteht durch K-Einfang des aktiven Eu-Isotops. Da diese KStrahlung mit derselben Halbwertzeit wie die $\beta$ _-Strahlung, nämlich 9,3 h abfällt, handelt es sich um einen dualen Zerfall. Für das Verzweigungsverhältnis erhält man aus den gemessenen Intensitäten nach Anbringen aller Korrektionen für Absorption und für dię innere Umwandlung der K-Strahlung (vergl. Ziff. 13): K-Einf./ $\beta$ Zer. $\approx 0,6$.

Die eigentliche $\gamma$-Strahlung des Eu $(9,3 \mathrm{~h})$ ist schon von Tyler (a.a.O.) spektroskopiert worden. Hiernach besteht sie aus drei Linien von 0,123 , 0,163 und 0,725 eMV. Aus unseren Messungen schätzt man die Zahl der $\gamma$-Quanten auf etwa 1,4 je $\beta$-Zerfall oder 2,3 je K-Einfang. Hiernach läßt sich nicht ohne weiteres entscheiden, wie weit die $\gamma$-Strahlen zu dem einen oder anderen Prozeß gehören; dazu wären Koinzidenzmessungen nötig. Es scheint aber, daß mindestens ein Teil der $\gamma$-Strahlung zum $\beta$-Zerfall gehört, denn, abgesehen von der großen Intensität der $\gamma$-Strahlen, sind auch in der Absorptionskurve der $\beta$-Strahlen (Abb. 7) gegen Ende der Reichweite zwei $\beta$-Gruppen mit verschiedener Grenzenergie zu erkennen.

Bei der langlebigen Aktivität war der durchdringende Strahlungsanteil $\mathrm{zu}$ schwach für eine so vollständige Prüfung, doch lassen auch hier die Blei-Absorptionsmessungen (Abb.6) mit großer Wahrscheinlichkeit auf einen entsprechenden dualen Zerfall schließen. Vor allem aber läßt die große Intensität der durchdringenden Strahlung im Verhältnis zur $\beta$-Strahlung kaum eine andere Deutung zu. Als Verzweigungsverhältnis ergibt sich K-Einf./B-Zerfall $\approx 12$ (Ziff. 13). Die Zahl der Kern- $\gamma$-Quanten ist von ähnlicher Größe wie die der K-Einfänge. Die $\gamma$-Strahlung wird also mindestens zum größeren Teil zum K-Einfang gehören.

Die Frage, ob die beiden aktiven Eu-Isotope $\mathrm{Eu}^{152,154}$ dual zerfallen, ist schon vorher des öfteren diskutiert, aber noch nicht entschieden wor$\operatorname{den}^{15}$. Für beide Isotope ist der duale Zerfall leicht verständlich, denn alle vier entstehenden Kerne sind stabil.

An Hand der Intensitätsverhältnisse läßt sich nun auch die bisher nicht bekannte Halbwertzeit des langlebigen Eu-Isotops abschätzen. Wie in

14 A. W. Ty le r, Physic. Rev. 56, 125 [1939]; K. F a jansu. D. Stew art, ebd. S. 625 .

${ }^{15} \mathrm{~K} . \mathrm{Fajans}$ u. D. W. Stew art ${ }^{14}$. 
Ziff. 13 gezeigt wird, ergibt sich durch Vergleich mit den Reinelementen Ho und Tm, daß der absolute Aktivierungsquerschnitt für die 9,3-h-Aktivität in der Nähe von $750.10^{-24} \mathrm{~cm}^{2}$ liegt (Tab.2). Der gesamte Aktivierungsquerschnitt für alle EuAktivitäten zusammen kann gleich dem von anderer Seite direkt gemessenen Absorptionsquerschnitt von etwa $3300.10^{-24}$ gesetzt werden (Tab. 2), da keine stabilen oder metastabilen Isotope gebildet werden. Daher bleibt für den Aktivierungsquerschnitt der langlebigen Aktivität 2550 $\cdot 10^{-24}$ übrig. Weiter ergaben die vorliegenden Messungen, daß nach kurzer Bestrahlung die $\beta$-Aktivität des 9,3-h-Zerfalls 14000-mal so stark ist wie die langlebige $\beta$-Aktivität. Rechnet man die jeweiligen K-Aktivitäten hinzu, so wird dieses Verhältnis 1800. Nun ist allgemein die Aktivität proportional dem Wirkungsquerschnitt und der reziproken Halbwertzeit. Daher gilt

$$
1800=\frac{750}{9,3}: \frac{2550}{\mathrm{~T}}
$$

wo $\mathrm{T}$ die gesuchte Halbwertzeit des langlebigen $\mathrm{Eu}$, in Stunden gemessen ist. So erhält man schließlich $\mathrm{T}=7 \pm 4 \mathrm{a}$. Die hohe Fehlergrenze ist durch die Unsicherheit der verschiedenen eingehenden Faktoren bedingt. Man erkennt, daß der große Absorptionsquerschnitt des $\mathrm{Eu}$ für thermische Neutronen zum überwiegenden Teil auf der Entstehung der langlebigen K-Aktivität beruht.

. Da die beiden stabilen Eu-Isotope Eu151, 153 gleich häufig sind, sind die Isotopenquerschnitte etwa 1500.10-24 für die 9,3-h-Aktivität, 5100 .10-24 für die 7-a-Aktivität. Eine sichere Zuordnung zu je einem der in Betracht kommenden Isotope $\mathrm{Eu}^{152,154}$ ist vorläufig nicht möglich. Die von Pool und $\mathrm{Qu}$ ill vorgenommene Zuordnung auf Grund eines angenommenen $(n, 2 n)$-Prozesses ist nicht stichhaltig (vergl. Ziff. 4).

8. Gadolinium. Ein $\mathrm{Gd}_{2} \mathrm{O}_{3}$-Präparat mit angeblich $0,2 \% \mathrm{~Tb}$ als einziger Verunreinigung ließ als sichere Aktivitäten nur die von $\mathrm{Eu}(9,3 \mathrm{~h}$ und $7 \mathrm{a})$, Sm ( $47 \mathrm{~h})$, Tb (73 d) und eine ungeklärte Aktivität von einigen Tagen erkennen. Immerhin widersprechen die Messungen nicht einer von P o ol u. Quill angegebenen schwachen Gd-Aktivität von $\mathrm{T}=17 \mathrm{~h}$.

9. Terbium. Es standen nur $21 \mathrm{mg}$ 80-proz.

16 W. B othe, Naturwiss. 31, 551 [1943].

17 S. Sugden, Nature [London] 135, 469 [1933]; G.Hevesy u. H.Levi ${ }^{13}$; M.L.Pool u. L.L.Quilli2.
$\mathrm{Tb}_{4} \mathrm{O}_{7}$ zur Verfügung. Die Aktivitätsanalyse mit thermischen Neutronen ergab Dy als Hauptverunreinigung. ,Sonst wurde nur die 73-d-Aktivität des $\mathrm{Tb}^{160}$ beobachtet, über die schon früher berichtet wurde ${ }^{16}$. Diese wurde zuerst an einem unreinen $\mathrm{Dy}_{2} \mathrm{O}_{3}$-Präparat gefunden, dessen $\mathrm{Tb}-\mathrm{Ge}$ halt sich hieraus zu etwa $6 \%$ ergab. Die Abfallskurven der beiden Präparate stimmten gut überein und ergaben als Halbwertzeit 73,5 $\pm 1 \mathrm{~d}$.

Die Absorptionskurven der beiden Präparate sind in Abb. 2 wiedergegeben. Wie man an der ebenfalls eingezeichneten Kurve für $\mathrm{Sc}^{46}$ (85 d) sieht, ist eine Verwechslung mit diesem Isotop ausgeschlossen. Die Form der Tb-Kurven läßt darauf schließen, daß die $\beta$ _-Strahlung aus mehreren Teilstrahlungen mit verschiedener Energiegrenze zusammengesetzt ist. Für die energiereichste Gruppe kann die Grenzenergie auf etwa 0,75 eMV geschätzt werden.

Der durchdringende Strahlungsanteil ist auffallend intensiv; er màcht sich naturgemäß bei dem dicken „Dy“-Präparat $\left(170 \mathrm{mg} / \mathrm{cm}^{2}\right)$ stärker bemerkbar als bei dem dünnen Tb-Präparat $(7 \mathrm{mg} /$ $\left.\mathrm{cm}^{2}\right)$. Hiernach entfallen mehrere $\gamma$-Quanten auf einen $\beta$-Zerfall. Es müssen also häufig angeregte Dy ${ }^{160}$-Zustände entstehen, die in mehreren Stufen in den Grundzustand übergehen. Hiermit ist die zusammengesetzte Struktur des $\beta$-Spektrums im Einklang. Außerdem besteht die Möglichkeit, daß der durchdringende Strahlungsanteil zum Teil (sicher nicht ganz) aus K-Strahlung besteht, die durch K-Einfang entsteht. Da Gd ${ }^{160}$ stabil ist, ist ein dualer $\beta$-K-Zerfall durchaus denkbar.

Für $\mathrm{Tb}^{160}$ ist von mehreren Seiten schon eine Halbwertzeit von 3,3 bis $3,9 \mathrm{~h}$ angegeben worden ${ }^{17}$. Diese Aktivität hätte hier wegen des hohen DyGehaltes unseres Tb-Präparates nicht beobachtet werden können. Ihre Existenz vorausgesetzt, müssen zwei isomere $\mathrm{Tb}^{160}$ angenommen werden.

10. Dysprosium. Auch das reinste der untersuchten $\mathrm{Dy}_{2} \mathrm{O}_{3}$-Präparate (,spektroskopisch reinst") ließ noch einen $\mathrm{Tb}$-Gehalt von $0,2 \%$ erkennen, daneben eine Spur Ho $(<0,1 \%)$. Nach Korrektion hierfür ergab sich ein rein exponentieller Abfall mit der Halbwertzeit $140 \pm 1,5$ min für das $Đ y^{165}$. Die höheren Literaturwerte (150 min und mehr) werden wohl auf einen längerlebigen Untergrund und zu kurze Meßdauer zurückzuführen $\operatorname{sein}^{18}$. Es

18 Die Herren W. Ri ezler und E. Fünfer teilten mir unabhängig mit, daß sie beide $\mathrm{T}=138 \mathrm{~min}$ gemessen haben, in Utbereinstimmung mit obigem Wert. 
ist allgemein $\mathrm{zu}$ beachten, daß die Hauptfehlerquellen bei der Bestimmung von Halbwertzeiten, nämlich langlebiger Untergrund und mangelndes Auflösungsvermögen bzw. Sättigungsmangel der Meßapparatur, beide im Sinne einer scheinbaren Verlängerung der Halbwertzeit wirken.

Die Absorptionskurve der Dy-Strahlung in Aluminium zeigt Abb. 7. Die Reichweite der $\beta$ _-Strahlen wurde nicht sehr genau bestimmt, sie entspricht den Literaturwerten für die Grenzenergie, die zwi-

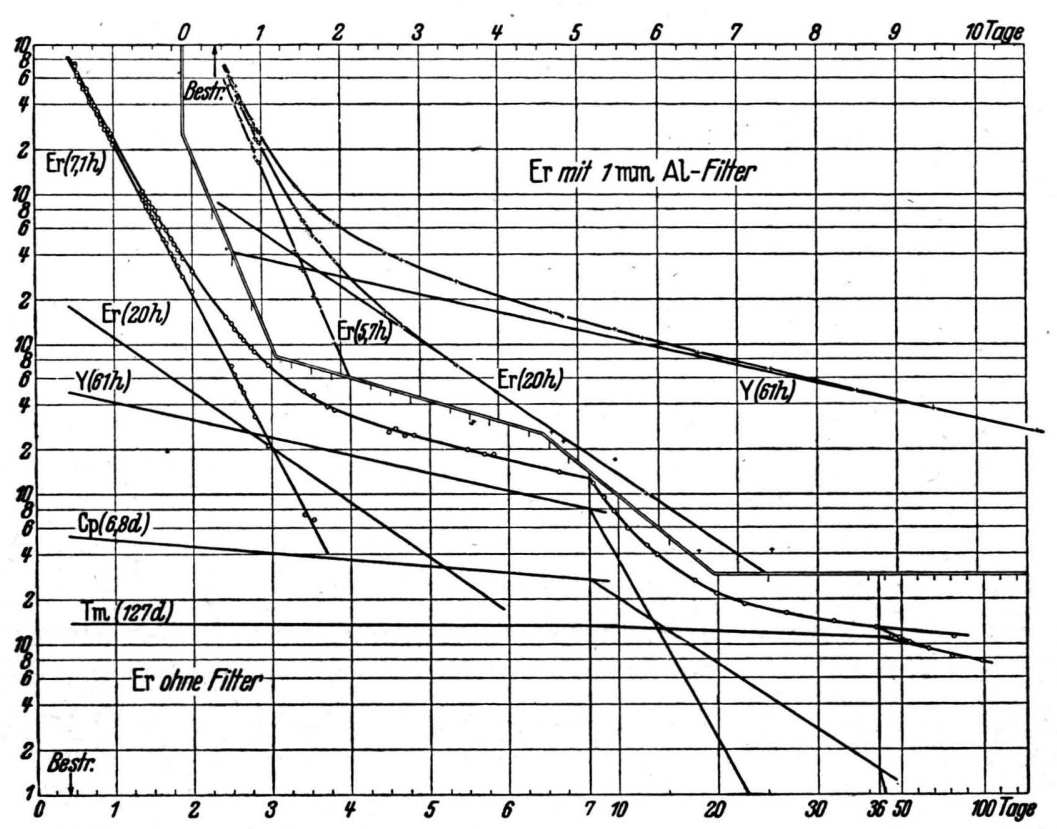

Abb. 8. Abfallskurven von Er (unrein).

schen 1,4 und 1,9 eMV liegen. Wenn neben der zu erwartenden Bremsstrahlung noch eine $\mathrm{K}$ - oder $\gamma$-Strahlung vorhanden ist, kann sie nur schwach sein.

11. Holmium. Das verwendete Präparat bestand aus $19 \mathrm{mg} \mathrm{Ho}_{2} \mathrm{O}_{3}$,reinst". $\left(=6,2 \mathrm{mg} / \mathrm{cm}^{2}\right)$. Es wurde die bekannte $\mathrm{Ho}^{166}$-Aktivität von $\mathrm{rd}$. $\mathrm{T}=30 \mathrm{~d}$ gemessen. Der Abfall wurde bis zu etwa $10^{-4} \mathrm{der}$ Anfangsaktivität verfolgt und ergab $\mathrm{T}=27,3$ $\pm 0,5 \mathrm{~h}$. Die längerlebige Restaktivität betrug nur rd. $3 \cdot 10^{-4}$.

Die Absorptionskurve der $\beta$-Strahlen zeigt Abb.1. Das $\beta$-Spektrum scheint einfach zu sein mit einer oberen Energiegrenze vion 1,8 eMV oder noch etwas höher (nach Hevesy u. Levi 1,6 eMV). Eine $\gamma$-Strahlung ist dementsprechend nicht vorhanden. Selbst die Bremsstrahlung macht sich bei einer Schwächung auf $0,04 \%$ noch nicht bemerkbar, da die Schicht dünn war und die austretenden $\beta$-Strahlen nur auf Aluminium trafen.

12. Erbium. Genaue Messungen an Er sind schwierig, weil dieses Element gewöhnlich mit anderen, stark aktivierbaren Erden verunreinigt ist, während die eigentlichen Er-Aktivitäten schwach sind. Für das reinste verfügbare $\mathrm{Er}_{2} \mathrm{O}_{3}$-Präparat war als einzige Verunreinigung $0,5 \%$ Yttrium angegeben. An diesem wurden Bestrahlungen in schwachen bis zu sehr starken Dosen vorgenommen. Abb. 8 zeigt die Abfallskurven für das unbedeckte Präparat sowie für Filterung mit $1 \mathrm{~mm} \mathrm{Al}$. Die letztere Kurve ist einfach zu analysieren. Es ergeben sich drei Komponenten, von denen die langlebigste die 61 - h - Aktivität des $\mathrm{Y}$ darstellt (Ziff. 1). Die beiden anderen Komponenten haben Halbwertzeiten von $20 \mathrm{~h}$ und 5,7 h. Die Abfallskurve des unbedeckten Präparates wurde länger verfolgt, weil sie sich als komplizierter erwies. $\mathrm{Zu}$ ihrer Darstellung sind mindestens 5 Komponenten nötig; die Halbwertzeiten sind: $\sim 4$ Monate, $7 \mathrm{~d}, 61 \mathrm{~h}, 20 \mathrm{~h}$ und $7,1 \mathrm{~h}$.

Die Absorptionsanalyse der längerlebigen Komponenten wurde wieder nach dem in I beschriebenen Verfahren durchgeführt. Die Komponente von 4 Monaten ist danach zweifellos Tm (127 d). Die 7-d-Komponente konnte an Hand der Absorptionskurve mit praktischer Sicherheit als $\mathrm{Cp}(6,8 \mathrm{~d})$ identifiziert werden. In der Tat haben Tm und Cp nach I so weiche $\beta$-Strahlungen, daß sie sich in der Abfallskurve mit $1 \mathrm{~mm}$ Al-Filter nicht mehr bemerkbar machen können. Dies ist wieder ein Beispiel für den Nutzen der Filterung. Die Absorptionskurve der 61-h-Komponente stimmte vollkommen mit der des $\mathrm{Y}$ überein. Die Gehalte an Y, Cp und Tm ergaben sich zu etwa 1,5; 0,3; 0,1\%. Für Ho fand sich kein Anzeichen.

Für das Er selbst bleiben danach die folgenden Halbwertzeiten übrig: $20 \pm 2 \mathrm{~h}$ (mit und ohne Filterung) und $7,1 \mathrm{~h}$ (ohne Filter) bzw. 5,7 h (mit $1 \mathrm{~mm}$ Al-Filter). Der Unterschied zwischen den letzten beiden Werten kann nicht durch eine Unsicherheit in der Zerlegung der Abfallskurven er- 
klärt werden. Es entsteht daher zunächst der Verdacht einer weiteren Verunreinigung. Unter den Erden ist aber keine Aktivität bekannt, deren Beimengung die Diskrepanz erklären könnte. Auch die geringe Menge $\mathrm{Cp}(3,7 \mathrm{~h})$, die mit dem $\mathrm{Cp}(6,8 \mathrm{~d})$ entstehen sollte, gibt keine Erklärung. Wenn also nicht noch irgend eine ausgefallene Verunreinigung des Er-Präparates vorliegt, muß man wohl schließen, daß die 6- bis 7-h-Aktivität des Er zusammengesetzt ist aus zwei Komponenten ähnlicher Halbwertzeit, von denen die kürzerlebige die durchdringendere Strahlung hat.

Die Absorptionskurven der beiden Er-Aktivitäten konnten nur durch eine ziemlich verwickelte Analyse gewonnen werden. Die Grenzenergien lassen sich daher nur schätzen auf $\sim 1,6 \mathrm{eMV}$ für den 6- bis 7-h-Körper, 0,6 eMV für den 20-hKörper. In beiden Fällen tritt auch eine $\gamma$-Strahlung auf, die beim 20-h-Körper besonders stark ist.

Frühere Untersuchungen haben eine Er-Aktivität von $12 \mathrm{~h}^{13}$ bzw. zwei von $5 \mathrm{~h}$ und $12 \mathrm{~h}^{12}$ ergeben. Hier konnte keine Andeutung einer 12-h-Aktivität gefunden werden. Es erscheint nicht ausgeschlossen, daß ein 12-h-Abfall vorgetäuscht werden kann, wenn die Messung nicht genügend weit ausgedehnt wird.

Eine weitere, früher ${ }^{11}$ angegebene, schwache 6-min-Aktivität des Er konnte auch hier nachgewiesen werden, und zwar mit etwa derselben Intensität. Sie wurde aber nicht genauer untersucht.

Für die mindestens drei Er-Isotope von $\mathrm{T}=6 \mathrm{~min}$, $6-7 \mathrm{~h}$ und $2 \theta \mathrm{h}$ stehen praktisch nur die Massenzahlen 169 und 171 zur Verfügung (Er ${ }^{165}$ ist als Positronenstrahler von 1,1 min bekannt ${ }^{12}$ ). Daher ist Isomerie möglich. Eine genauere Zuordnung ist vorläufig nicht möglich. Vielleicht gehört aber auch eine der Halbwertzeiten zu $\mathrm{Tm}^{171}$, das aus einem kurzlebigen $\operatorname{Er}^{171}$ entstehen könnte.

13. Intensitätsverhältnisse. Zum Schluß seien noch einige praktisch und theoretisch interessante Intensitätsangaben gemacht. Man kann aus den Intensitäten, die man mit einer bestimmten Bestrahlungsstärke und -dauer erhält, auf die relativen Wirkungsquerschnitte der Aktivierung schließen. Hierzu ist allerdings eine Reihe von Umrechnungen nötig, die im vorliegenden Falle meist nicht sehr genau durchzuführen sind:

a) Die Strahlen des aktivierten Präparates werden z. Tl. schon im Präparat selbst absorbiert. Ist $\mu$ der Absorptionskoffizient, d die Dicke des (homo-

10 Erscheint demnächst. gen durchaktivierten) Präparates, so kommt von den einseitig emittierten Strahlen nur der Bruchteil $\left(1-\mathrm{e}^{-\mu \mathrm{d}}\right) / \mu \mathrm{d}$ zum Austritt.Für die $\beta$-Strahlen muß $\mu$ aus der gemessenen Absorptionskurve entnommen werden, wobei aber etwaige sehr energiearme $\beta$-Komponenten nicht miterfaßt werden. Für die K-Strahlungen können die direkten Absorptionsmessungen mit selektiven Filtern (Ziff. 6 u. 7) benutzt werden.

b) Auf dem Wege in das Zählrohr haben die $\beta$-Strahlen noch eine Cellophanfolie von $4 \mathrm{mg} / \mathrm{cm}^{2}$ und die Zählrohrwand von $27 \mathrm{mg} / \mathrm{cm}^{2}$ zu durchdringen. Die entsprechende Absorption läßt sich mit dem erwähnten $\mu$-Wert berücksichtigen, wiederum mit dem Vorbehalt, daß keine sehr energiearmen Komponenten vorhanden sind. Die K-Strahlen mußten hinter 1 bis $3 \mathrm{~mm} \mathrm{Al}$ gemessen werden, um die $\beta$-Strahlen wegzufiltern, und wurden dabei ebenfalls merklich absorbiert.

c) Bei den K- und $\gamma$-Strahlen ist die Ansprechwahrscheinlichkeit des Al-Zählrohres zu berücksichtigen. H. M aier-Leibnitz hat in neuen Versuchen $^{19}$ die hierfür von N o r l in ${ }^{20}$ gegebene Kurve im wesentlichen bestätigt und nach kleineren Quantenenergien fortgesetzt. Für die hier vorkommenden K-Strahlungen gilt etwa ein Wert von $8 \cdot 10^{-4}$. Ferner ist $\mathrm{zu}$ berücksichtigen, daß rd. $15 \%$ der K-Strahlung durch innere Umwandlung (A u ger Effekt) verloren gehen.

d) Wenn das Präparat die thermischen Neutronen stark absorbiert, senkt es die Neutronendichte, die an seinem Ort bei der Bestrahlung in Paraffin herrscht; die Aktivierung fällt daher relativ zu anderen, schwach absorbierenden Präparaten zu klein aus. Ist dieser Effekt nicht sehr groß, so kann er an Hand früher mitgeteilter Berechnungen und Messungen $^{21}$ berücksichtigt werden. Für die besonders stark absorbierenden Elemente wurden einige neue Vergleichsmessungen mit dicken und dünnen Präparaten gemacht. Bei einer Oxydmenge von $0,5 \mathrm{~g}$ auf einer Kreisfläche von $2 \mathrm{~cm}$ Durchmesser wird Dy etwa 2-mal, Eu etwa 3-mal, Sm etwa 4-mal zu schwach aktiviert.

In Tab. 2 enthalten die Spalten 2 und 3 Relativwerte der Aktivitäten, die unmittelbar nach einer kurzen Bestrahlung mit thermischen Neutronen mit einem Al-Zählrohr von 0,1 mm Wand gemessen wurden. Spalte 2 gilt für Präparate, die $0,5 \mathrm{~g}$ Oxyd auf einer Kreisfläche von $2 \mathrm{~cm}$ Durchmesser ent-

20 F. Norling ${ }^{8}$.

21 W. B othe, Z. Physik 120, 437 [1943]. 


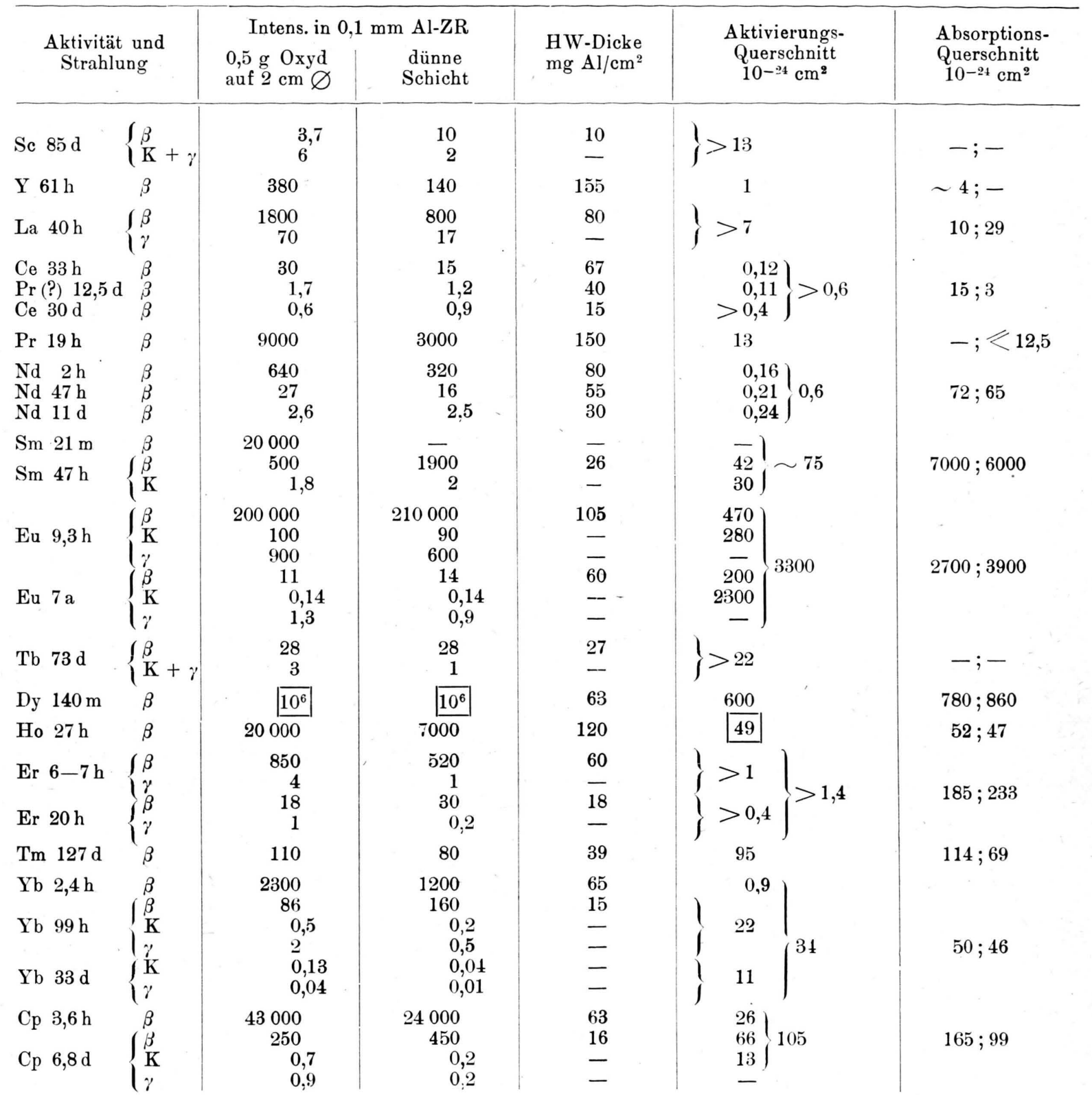

Tab. 2. Intensitätsangaben für die Bestrahlung seltener Erden mit thermischen Neutronen entstehende Aktivität.

halten. Spalte 3 gilt für unendlich dünne Oxydpräparate von gleicher Masse. Spalte 3 ergibt sich aus Spalte 2 durch Abschätzung der Einflüsse a) und d). In beiden Spalten ist die Dy-Aktivität $=10^{6}$ gesetzt. Schon von diesen Zahlen darf keine allzu große Genauigkeit erwartet werden, zumal verschiedene und z. Tl. nicht sehr konstante Strahlen-

${ }^{22}$ In einigen Fällen war Hr. Ri e z l e r so freundlich, die relativen Dosen an einem mitaktivierten Stückchen Tantalblech zu messen. Die Aktivität des entstehenden quellen verwendet werden mußten ${ }^{22}$. Die Genauigkeit dürfte aber für viele Analysenzwecke ausreichen. Für die Umrechnung auf andere Meßbedingungen und zur einfachen Kennzeichnung des Durchdringungsvermögens sind in Spalte 4 die Halbwertdicken der $\beta$-Strahlen in Aluminium aufgeführt.

$\mathrm{Ta}^{182}\left(10^{0} \mathrm{~d}\right)$ ist noch bei mehrtägiger Bestrahlung proportional zur Dosis. Das Riezlersche Verfahren hat sich gut bewährt. 
Spalte 5 enthält die Wirkungsquerschnitte der Aktivierung normaler Isotopengemische. Hierfür wurden die gemessenen Aktivitäten auf eine bestimmte kurzzeitige Bestrahlungsdosis und auf 1 Mol umgerechnet, dann korrigiert für alle oben erwähnten Einflüsse und mit der Halbwertzeit multiplizįert. Schließlich wurde noch ein Normierungsfaktor angebracht, der so bemessen ist, daß die Zahlen als angenäherte Absolutwerte der Aktivierungsquerschnitte angesehen werden können. Dieser Faktor wurde folgendermaßen bestimmt: In Spalte 6 der Tabelle sind die direkt gemessenen $A b$ sorptions-Querschnitte für thermische Neutronen aufgeführt ${ }^{23}$. Für Ho und Tm muß nun der $A b$ sorptionsquerschnitt mit dem $\beta$-Aktivierungsquerschnitt übereinstimmen, da beides Reinelemente sind, und da die daraus entstehenden aktiven Isotope keine merkliche K- und $\gamma$-Strahlung aussenden und anscheinend auch keine Isomere besitzen. Hieraus ergibt sich der Normierungsfaktor, und zwar befriedigenderweise für beide Elemente übereinstimmend. Beim Ce wurde so gerechnet, als ob die 12,5-d-Aktivität primär, und nicht erst aus der 33-h-Aktivität entstünde (Ziff. 3).

In einigen Fällen konnten nur untere Grenzen für den Aktivierungsquerschnitt angegeben werden, weil die Stärke der $\gamma$-Strahlung vermuten läßt, daß das $\beta$-Spektrum zusammengesetzt ist, und da $\beta$ weichere $\beta$-Komponenten der Messung entgingen. Beim $\mathrm{Yb}(99 \mathrm{~h})$ konnte hierfür eine Korrektion

23 Die erste Zahl stammt jeweils von H. B o m ke u. H. Reddemann (Z. Physik 120, 56 [1942]), die zweite von W. Riezler (unveröffentlicht); vergl. den Bericht von K. Diebner, W. Herrmann u. E. Grassmann, Physik. Z. 43, 440 [1942]. von $+40 \%$ geschätzt und angebracht werden (vergl. I). Der Vergleich mit älteren Angaben von Hevesy und Levi (a. a. O.) zeigt nicht durchweg Übereinstimmung, insbesondere ist $\mathrm{Cp}(6,8 \mathrm{~d})$ relativ zu Dy nach unseren Messungen rund 10-mal stärker aktiv als nach Heves y und Levi. Bemerkenswert ist noch, daß sich aus dem $\mathrm{K}$-strahlenden $\mathrm{Yb}^{169}$ (33 d) ein sehr hoher Isotopen-Querschnitt des $\mathrm{Yb}^{\mathbf{1 6 8}}$ ergibt. Da dessen Isotopènhäufigkeit nur $0,06 \%$ beträgt, ist der Isotopenquerschnitt rd. $20000 \cdot 10^{-24}$, das ist einer der größten bekannten Wirkungsquerschnitte.

Interessant ist schließlich noch ein Vergleich der Spalten 5 und 6 der Tab. 2. Bei den Reinelementen (Sc, Y, La, Pr, Tb, Ho, Tm) und beim Ce sollten Aktivierungs- und Absorptionsquerschnitte übereinstimmen, weil durch den $(n, \gamma)$-Prozeß nur aktive Isotope entstehen können. Dasselbe gilt für Cp, wo nur der Prozeß $\mathrm{Cp}^{175}(\mathrm{n}, \gamma) \mathrm{Cp}^{176}$ zu einem (praktisch) stabilen Isotop führen könnte; dieser Prozeß wird aber wegen des großen Spinunterschiedes nicht auftreten, statt dessen entsteht das Isomer ${ }^{*} \mathrm{Cp}^{176}$ (6,8 d). So weit die, allerdings noch recht lückenhaften, Messungen reichen, sind in der Tat in'diesen Fällen die Spalten 5 und 6 nicht im Widerspruch miteinander. Bei Eu wurde die ebenfalls zu verlangende UUbereinstimmung durch die Wahl der längeren Halbwertzeit hergestellt (Ziffer 7). Bei den übrigen Elementen gibt es benachbarte stabile Isotope, so daß $(n, \gamma)$-Prozesse ohne Aktivierung vorkommen können. Daher sind hier die Absorptionsquerschnitte z. Tl. erheblich größer als die Aktivierungsquerschnitte.

Den Herren Gentner, Ma urer und Riezler habe ich für Hilfe bei den Bestrahlungen zu danken. 